\title{
Women's Health Care: the Potential of Long-Chain Omega-3 Polyunsaturated Fatty Acids
}

\section{Hunt $\mathbf{W}^{*}$ and McManus $A$}

The Centre of Excellence for Science Seafood and Health, Curtin University, Australia

\begin{abstract}
Health care requirements are challenged by longer life expectancies with the fastest growing age group being women aged 85 years and older. The health care requirements for older women require further investigation with clinical trials seeking to represent this growing proportion of our population. Gender specific and general health care requirements for women over their lifespan need to be aimed at prolonging health adjusted life expectancy.

Health care utilization has been linked to exposure to printed and online health information although which is the causal factor is unknown. Health literacy remains a concern in the transmission of health information especially with regard to complex messages such as the many health benefits associated with marine sourced long-chain omega-3 polyunsaturated fatty acids. These health benefits include: all-cause morbidity, coronary heart disease, cardiovascular disease, mental health including depression, dementia and Alzheimer's disease, polycystic ovarian syndrome, dysmenorrhea and infant cognitive development. In many cases prevention is possible or at the very least a delay in onset of disease. Depression remains the single greatest contributor to the burden of disease in women in low-, medium- and high-income countries.
\end{abstract}

Quality of womens' health care throughout the lifespan and targeted health communications including those around diet has the ability to greatly influence health adjusted life expectancy.

Keywords: Women; Health care; Health outcomes; Diet; Omega-3 fatty acids

\section{Introduction}

Health care has substantially contributed to increased life expectancy in Australia and many other developed countries [1]. Health adjusted life expectancy for females and males, born in 2003 in Australia, was 75.2 and 70.6 years respectively [2]. Among the health and health care challenges identified by the Australian Government are the needs to invest more in the health of our children and to address changes in the disease pattern of our aging population and the associated increase in chronic illness [3]. These health care challenges, and others, span our lifetime. This paper provides a brief review of the need for quality health care for women throughout their lifespan and the potential influence dietary interventions.

\section{The Case for Omega-3 Polyunsaturated Fatty Acids in Womens' Health Care}

Despite decreases in childhood mortality, conditions such as low birth weight, increased incidence of obesity and diabetes, asthma, allergies, developmental disorders, autism, mental health disorders and behavioural disturbances influence life expectancy and quality of life [3]. Children from the rural environment and those from the lowest socioeconomic areas are most at risk [4]. The World Health Organisation (WHO) acknowledges that children who are disadvantaged either socioeconomically or nutritionally are never able to catch up to affluent and healthy children [5]. Healthy children, able to grow and meet their full educational, social and career potential are a valuable economic resource placing less burden on the health care system and associated budgets; these children can become tomorrow's inter generationally competent parents [4].

Socioeconomic discrepancies are not alleviated by a universal health system such as Australia's with health adjusted life expectancy ranging from 71.2 to 75.5 years across socioeconomic quintiles [1,2]. Mortality and socioeconomic status are thought to be related as higher socioeconomic status is commonly associated with greater access to knowledge, money, power, prestige and social connections; these resources allow people to avoid diseases and to minimise negative outcomes [1]. Above and beyond the motivation to seek out and access health information is the concept of health literacy. It is estimated, depending on the measurement tools utilized, that between $6.8 \%$ and $26.0 \%$ of Australian adults have suboptimal health literacy levels [6].

Exposure to online and printed health information has been positively correlated with increased health care utilization; it remains unclear however whether health care utilization or information exposure is the causal factor [7]. One complex message that is little understood is the benefits to health associated with the consumption of fish, seafood and marine sourced omega-3 fatty acids [8]. All omega-3 fatty acids are not equally efficacious to health; the great majority of evidence is associated with the marine sourced long-chain polyunsaturated omega-3 fatty acids, docosahexaenoic acid and eicosapentaenoic acid, rather than intakes of the essential precursor fatty acids [8].

Lower amounts of docosahexaenoic acid in breastfeeding mothers' milk as well as a diet low in seafood consumption have been linked to higher incidence of postpartum depression [9]. The disease burden potentially attributable to long-chain omega-3 fatty acid deficiency based on worldwide diversity is $42.5 \%$ of female coronary heart disease mortality; $31.1 \%$ of female stroke mortality; $39.1 \%$ of female cardiovascular disease mortality; $31.5 \%$ of total female mortality; and $65.5 \%$ of postpartum depression [10]. Despite the importance of

*Corresponding authors: Dr. Wendy Hunt, Deputy Director, Senior Food Scientist, The Centre of Excellence for Science Seafood and Health, Curtin University, 7 Parker Place, Technology Park, 6102, Australia, Tel: +61 423972 993; Fax: +61 8 9266 2508; E-mail: W.Hunt@Curtin.edu.au

Received December 23, 2013; Accepted January 24, 2014; Published January 28,2014

Citation: Hunt W, McManus A (2014) Women's Health Care: the Potential of LongChain Omega-3 Polyunsaturated Fatty Acids. J Women's Health Care 3: 142. doi:10.4172/2167-0420.1000142

Copyright: $\odot 2014$ Hunt W, et al. This is an open-access article distributed under the terms of the Creative Commons Attribution License, which permits unrestricted use, distribution, and reproduction in any medium, provided the original author and source are credited. 
omega-3 fatty acid consumption to both maternal health and foetal neurological development, it has become apparent that although pregnant women actively seek information about nutritional changes, health-care services have not been providing adequate information about the importance of consuming omega-3 fatty acids during pregnancy [11].

Women have health care requirements for gender specific conditions. Based on research carried out on a representative sample of American women, female specific conditions and disorders affect more than $20 \%$ of women each year [12]. The researchers note pregnancy and pregnancy related conditions were the most prominent female-specific reason women aged 14-29 years sought health care and the second most common for women aged 30-44 years. The largest health expenditures were noted for pregnancy related, gynaecologic, menopausal, benign breast and female cancer conditions. Despite significant gender-specific conditions, depression is responsible for the greatest burden of disease in women in low-, middle- and high- income countries highlighting the need for minimisation strategies [5].

Intake of marine sourced long-chain omega-3 fatty acids have been positively associated with women's health with regard to all-cause morbidity, coronary heart disease, cardiovascular disease, mental health including depression, dementia and Alzheimer's disease, polycystic ovarian syndrome, dysmenorrhea and infant cognitive development [13]. Despite evidence surrounding the benefits of omega-3 fatty acid consumption, particularly increasing the ratios of omega- 3 to omega- 6 fatty acids in the diet, a whole of diet approach is preferred.

A Western dietary pattern is described as omega-3 fatty acid (docosahexaenoic acid in particular) deficient [14]. In evolutionary terms, decreased consumption of fish and increased consumption of foods high in omega- 6 fatty acids has changed our dietary ratio from roughly 1-2:1 as hunter gatherers to 20-30:1 in a Western diet [15]. Contrastingly, conformance with a Mediterranean diet, that is a diet high in fruit, vegetables, fish, nuts, legumes and grains and low in red meat, has been associated with a reduction in risk of death from all causes including cancer and cardiovascular disease [16].

The quality of women's health care and the importance of appropriately targeted health communications gains increasing impetus. The fastest growing age group are the over 85 year olds, the majority of which are female [17]. In contrast to our aging population is that geriatric medicine is underrepresented in many medical schools; additionally although cancer and heart disease are the leading causes of death in age groups 65 years and older, older adults are frequently underrepresented in clinical trials [17]. The health care needs of this group both in prevention and treatment require further investigation.

\section{Potential for Long-Chain Omega-3 Polyunsaturated Fatty Acids in Womens' Health Care}

The mechanisms for marine sourced long-chain omega- 3 fatty acids, and increasing dietary ratios of omega- 3 to omega- 6 fatty acids, continue to be explored. The neuroprotective role of docosahexaenoic acid is explained by multiple mechanisms when reviewed with emerging research data adding to a growing body of evidence [14]:

1. Anti-inflammatory action through competition with omega-6 arachidonic acid for esterification into phospholipids; reducing arachidonic acid and subsequent reduction of metabolites;

2. Insulin / trophic factor activity to resulting in down-regulation of pro-apoptotic regulators influencing cell death;
3. Increases in brain-derived neurotrophic factor which is typically found to be depleted in the hippocampus of patients with Alzheimer's disease;

4. Antioxidant activity and reduction of oxidative damage;

5. Lipoxidase metabolite of docosahexaenoic acid increasing activity of anti-apoptotic regulators and reducing activity of pro-apoptotic regulators influencing cell death;

6. Other neuroprotective functions including promotion of neurogenesis, increased glucose transport influence on blood flow and glucose utilization, improvement in the fluidity of synaptic membranes, benefits to signal, and transduction pathways.

The potential benefits of dietary intervention approaches and or supplementation with marine sourced omega-3 fatty acids are immense. Within a healthy dietary pattern, fish and seafood offer many highly bioavailable nutrients in addition to their omega- 3 content. Interventions for prevention of diseases including cardiovascular disease, dementia and Alzheimer's disease alone are needed as omega-3 fatty acids have the advantage of being low in cost yet offering longterm safety [14]. Additionally, there is considerable potential for use of dietary interventions as complementary treatments for many chronic diseases.

\section{Conclusion}

The use of multifaceted approaches to prolong healthy life expectancy becomes increasingly important as our population ages. Attention to diet has the potential to greatly reduce the burden of disease in women. Optimal omega- 3 fatty acid status has the ability to prevent or significantly delay the onset of chronic conditions including coronary heart disease, dementia and Alzheimer's disease.

\section{References}

1. Korda RJ, Butler JR, Clements MS, Kunitz SJ (2007) Differential impacts of health care in Australia: trend analysis of socioeconomic inequalities in avoidable mortality. Int J Epidemiol 36: 157-165.

2. Begg S, Vos T, Barker B, Stevenson C, Stanley L, et al. (2007) The burden of disease and injury in Australia 2003. Australian Institute of Health and Welfare (AlHW).

3. Armstrong BK, Gillespie JA, Leeder SR, Rubin GL, Russell LM (2007) Challenges in health and health care for Australia. Med J Aust 187: 485-489.

4. Stanley F. The linking of records and analysis of population data for epidemiological and public health research. The Griffith Lecture 2005.

5. World Health Organisation (2008) The Global Burden of Disease. WHO, Geneva.

6. Barber MN, Staples M, Osborne RH, Clerehan R, Elder C, et al. (2009) Up to a quarter of the Australian population may have suboptimal health literacy depending upon the measurement tool: results from a population based survey. Health Promotion International 24: 252-261.

7. Nicholson W, Gardner B, Grason HA, Powe NR (2005) The association between women's health information use and health care visits. Womens Health Issues 15: 240-248.

8. McManus A, Merga M, Newton W (2011) Omega-3 fatty acids. What consumers need to know. Appetite 57: 80-83.

9. Hibbeln JR (2002) Seafood consumption, the DHA content of mothers' milk and prevalence rates of postpartum depression: a cross-national, ecological analysis. J Affect Disord 69: 15-29.

10. Hibbeln JR, Nieminen LR, Blasbalg TL, Riggs JA, Lands WE (2006) Healthy intakes of n-3 and n-6 fatty acids: estimations considering worldwide diversity. Am J Clin Nutr 83: 1483S-1493S. 
Citation: Hunt W, McManus A (2014) Women's Health Care: the Potential of Long-Chain Omega-3 Polyunsaturated Fatty Acids. J Women's Health Care 3: 142. doi:10.4172/2167-0420.1000142

11. Sinikovic DS, Yeatman HR, Cameron D, Meyer BJ (2009) Women's awareness of the importance of long-chain omega-3 polyunsaturated fatty acid consumption during pregnancy: knowledge of risks, benefits and information accessibility. Public Health Nutr 12: 562-569.

12. Kjerulff KH, Frick KD, Rhoades JA, Hollenbeak CS (2007) The cost of being a woman: a national study of health care utilization and expenditures for femalespecific conditions. Womens Health Issues 17: 13-21.

13. McManus A, Hunt W (2013) Consumption of Omega-3 Fatty Acids and Women's Health. OA Women's Health 1: 11.

14. Cole GM, Ma QL, Frautschy SA (2009) Omega-3 fatty acids and dementia. Prostaglandins Leukot Essent Fatty Acids 81: 213-221.
15. Yashodhara BM, Umakanth S, Pappachan JM, Bhat SK, Kamath R, et al. (2009) Omega-3 fatty acids: a comprehensive review of their role in health and disease. Postgrad Med J 85: 84-90.

16. Mitrou PN, Kipnis V, ThiébautAC, Reedy J, Subar AF, et al. (2007) Mediterranean dietary pattern and prediction of all-cause mortality in a US population: results from the NIH-AARP Diet and Health Study. Arch Intern Med 167: 2461-2468.

17. Kosiak B, Sangl J, Correa-de-Araujo R (2006) Quality of health care for older women: what do we know? Womens Health Issues 16: 89-99.
Citation: Hunt W, McManus A (2014) Women's Health Care: the Potential of Long-Chain Omega-3 Polyunsaturated Fatty Acids. J Women's Health Care 3: 142. doi:10.4172/2167-0420.1000142
Submit your next manuscript and get advantages of OMICS Group submissions

\section{Unique features:}

ublishing.

User friendly/feasible website-translation of your paper to 50 world's leading language Audio Version of published paper

Digital articles to share and explore

\section{Special features:}

300 Open Access Journals

25,000 editorial team

21 days rapid review process

Quality and quick editorial, review and publication processing

Indexing at PubMed (partial), Scopus, EBSCO, Index Copernicus and Google Scholar etc

Sharing Option: Social Networking Enabled

Authors, Reviewers and Editors rewarded with online Scientific Credits

Better discount for your subsequent articles

Submit your manuscript at: http://www.omicsonline.org/submissio 